\title{
Treatment of Supraorbital Neuralgia Using Ultrasound-Guided Radiofrequency Thermocoagulation of the Supraorbital Nerve: A Retrospective Study
}

This article was published in the following Dove Press journal:

Journal of Pain Research

Hao Ren

Ying Shen*

Fang Luo*

Department of Pain Management, Beijing Tiantan Hospital, Capital Medical

University, Beijing, People's Republic of China

*These authors contributed equally to this work
Correspondence: Fang Luo; Ying Shen Department of Pain Management, Beijing Tiantan Hospital, Capital Medical University, No. 119 West Road, South 4th Ring Road, Fengtai District, Beijing 100050, People's Republic of China Tel +86 I36II326978; +86 I86/079|6I7 Email luofangwt@yahoo.com; shenying1937@hotmail.com
Purpose: There is no standard clinical treatment protocol for supraorbital neuralgia patients who respond poorly to conservative treatment. Radiofrequency thermocoagulation, a neurologically damaging procedure, is a treatment for supraorbital neuralgia. However, assessments of its longterm efficacy are lacking. Thus, this study aimed to evaluate the long-term efficacy and safety of ultrasound-guided radiofrequency thermocoagulation for treating supraorbital neuralgia.

Patients and Methods: We retrospectively reviewed our clinical database for supraorbital neuralgia patients who underwent an ultrasound-guided radiofrequency thermocoagulation procedure. Demographic data and baseline characteristics, time of onset, postoperative pain intensity, time of recurrence, subsequent treatment, complications and side effects were collected and analysed. The Kaplan-Meier estimator was used to determine recurrence-free survival.

Results: A total of 53 supraorbital neuralgia patients were included in this study. All patients experienced complete pain relief within one month. The median follow-up time of the 53 patients was 36.0 months (IQR, 12.0-72.0 months). A total of 13 patients experienced pain recurrence, with a median recurrence-free time of 97 months according to the Kaplan-Meier estimator. The cumulative proportion of recurrence-free survival was $96.2 \%$ at 12 months, $88.4 \%$ at 24 months, $82.7 \%$ at 36 months, $70.0 \%$ at 48 months, $66.3 \%$ at 60 months, and $49.7 \%$ at 97 months. All but one patient with recurrent pain underwent a second or third radiofrequency thermocoagulation procedure and achieved complete pain relief. Numbness of supraorbital nerve innervation occurred in all patients. However, the numbness scores gradually decreased over time.

Conclusion: Ultrasound-guided radiofrequency thermocoagulation is a safe, effective treatment for supraorbital neuralgia patients who respond poorly to conservative treatments. These patients attained excellent long-term pain relief with a gradual reduction in numbness. Keywords: supraorbital neuralgia, radiofrequency thermocoagulation, long-term efficacy, survival analysis

\section{Introduction}

The supraorbital nerve is a purely sensory branch of the frontal nerve that comes from the ophthalmic division of the trigeminal nerve. This nerve traverses the supraorbital notch/foramen and divides into the medial and lateral branches. The supraorbital nerve, accompanied by the supraorbital artery, supplies the conjunctiva, upper eyelid, forehead, and anterior scalp up to the lambdoid suture. ${ }^{1}$ 
Supraorbital neuralgia is an uncommon form of neuropathic pain. An epidemiological survey of 1838 community populations showed an incidence of $0.65 \%{ }^{2}$ In the 2nd edition of the International Classification of Headache Disorders (ICHD-2), ${ }^{3}$ the diagnostic criteria for supraorbital neuralgia are as follows: 1. paroxysmal or constant pain in the region of the supraorbital notch and the medial aspect of the forehead in the area supplied by the supraorbital nerve, 2. tenderness over the nerve in the supraorbital notch, and 3. pain abolished by local anaesthetic blockade or ablation of the supraorbital nerve. However, in the ICHD- $3,{ }^{4}$ supraorbital neuralgia is no longer classified as an independent disease. In the International Classification of Diseases 11th Revision (ICD-11), ${ }^{5}$ supraorbital neuralgia is classified as 8 A85. Painful cranial neuropathies or other facial pains. Trauma or compression of the supraorbital nerve is the main aetiology of supraorbital neuralgia. ${ }^{6}$ Supraorbital neuralgia can be induced by direct trauma or repetitive microtrauma from wearing overly tight swim goggles. ${ }^{7-9}$

Except in cases of recovery without oral medical intervention, especially those involving post-traumatic supraorbital neuralgia, ${ }^{10,11}$ patients with supraorbital neuralgia should first be treated with drugs such as carbamazepine, gabapentin, and pregabalin. ${ }^{7,12-14}$ For some patients, supraorbital nerve block using local anaesthetics with or without glucocorticoids can provide pain relief, but multiple injections are often required. ${ }^{8,13,15}$ For patients with supraorbital neuralgia who have failed to respond to conservative treatments, some neurologically damaging procedures (chemical neurolysis, ${ }^{16}$ surgical resection of the supraorbital nerve, ${ }^{17}$ cryoneuroablation, ${ }^{18}$ and radiofrequency thermocoagulation ${ }^{19}$ ) or non-destructive procedures (surgical decompression of the supraorbital nerve, ${ }^{20}$ peripheral nerve stimulation, ${ }^{21}$ and pulsed radiofrequency ${ }^{22}$ ) may be alternative treatments. However, clinical research data are quite rare, and there is no general agreement regarding the standard treatment protocol for supraorbital neuralgia.

Chemical neurolytic techniques have been developed to promote longer effects compared to those of nerve blocks. However, these techniques carry a significant risk of skin sloughing and neuritis. ${ }^{23}$ Compared with percutaneous puncture techniques, surgical decompression or resection of the supraorbital nerve and supraorbital nerve stimulation are more traumatic. Peripheral nerve stimulation can also result in more complications, including skin erosion, breakdown of the anchoring site, infection and lead migration, and it can also present a heavy economic burden for patients. A series of studies have reported that pulsed radiofrequency, a percutaneous minimally invasive treatment, can provide pain relief for patients without the side effects of neural damage. ${ }^{22,24-26}$ Among these reports, our previous study demonstrated that pulsed radiofrequency for the treatment of refractory supraorbital neuralgia had effective rates of $77 \%$ and $50 \%$ at one month and two years postoperatively, respectively. ${ }^{22}$ Although side effects are rare after pulsed radiofrequency treatment, the effective rate of pulsed radiofrequency still needs improvement.

Unlike pulsed radiofrequency, radiofrequency thermocoagulation is a neurologically damaging procedure that is often used to treat trigeminal neuralgia. ${ }^{27}$ Radiofrequency thermocoagulation has been reported as a treatment for supraorbital neuralgia; ${ }^{13,19}$ however, there is little literature on this subject. As a percutaneous interventional technique, radiofrequency thermocoagulation for supraorbital neuralgia is easier to perform than surgical decompression or resection and peripheral nerve stimulation; additionally, it involves less trauma and a lower cost. However, assessments of its long-term efficacy are lacking. This study aims to evaluate the long-term efficacy and safety of ultrasound-guided radiofrequency thermocoagulation for the treatment of supraorbital neuralgia (unresponsive to conservative treatment) in a relatively large case series.

\section{Materials and Methods}

\section{Patients}

This study was approved by the Institutional Review Board of Beijing Tiantan Hospital affiliated with Capital Medical University and was conducted in compliance with the current version of the Declaration of Helsinki. This retrospective study involved no more than minimal risk to subjects. The data included in the analysis were all de-identified. The waiver of consent did not adversely affect the subjects' welfare and rights. The Institutional Review Board of Beijing Tiantan Hospital approved the application for a waiver of informed consent for this study. We retrospectively reviewed our clinical database for supraorbital neuralgia patients who underwent a radiofrequency thermocoagulation procedure at the Department of Pain Management, Beijing Tiantan Hospital between January 2007 and April 2018. The available follow-up data ended on May 2019. Patients who met the following criteria were eligible: 1 . age $>18$ years; 2 . diagnosis of supraorbital 
neuralgia according to the diagnostic criteria of the International Classification of Headache Disorders; 3. radiofrequency thermocoagulation treatment of the supraorbital nerve for supraorbital neuralgia; and 4. available follow-up data for at least one year after the procedure. The exclusion criteria were 1. supraorbital neuralgia caused by an intracranial mass and 2. incomplete medical records.

\section{Operative Technique}

The ultrasound-guided radiofrequency thermocoagulation procedure in our institution was performed as follows. Noninvasive blood pressure (taken at five-minute intervals), blood oxygen saturation and electrocardiograms were recorded routinely. The return plate of the pain management generator (PMG-230, Baylis Medical, Inc., Canada) was attached to the back skin of the patient. After the skin was prepared with an antiseptic solution, aseptic drapes were applied to the superciliary arch. Supraorbital nerve block was performed with $1 \%$ lidocaine. The high frequency linear transducer of the ultrasound device (SonoSite, Inc., USA) was prepared with a sterile sheath and aseptic ultrasound gel. The ultrasound probe was moved from the forehead caudally to locate the supraorbital notch, which is a small groove on the bony surface (Figure 1A). A 21-gauge radiofrequency treatment trocar needle with a 2-mm noninsulated tip (PMF-21-50-2, Baylis Medical Inc.) was used to puncture the surface and was moved towards the supraorbital notch under ultrasound guidance using an in-plane or out-of-plane approach (Figure 1B). When the trocar needle was inserted precisely into the supraorbital notch and negative aspiration for blood was confirmed, the stylet was removed, and the radiofrequency treatment electrode (PMK-21-50, Baylis Medical, Inc.) was inserted into the trocar. The parameters of radiofrequency thermocoagulation were set as $60^{\circ} \mathrm{C}, 75 \mathrm{~s} ; 65^{\circ} \mathrm{C}, 75 \mathrm{~s} ; 70^{\circ} \mathrm{C}, 75 \mathrm{~s} ; 75^{\circ} \mathrm{C}, 75 \mathrm{~s}$; and $80{ }^{\circ} \mathrm{C}, 75 .^{28-31}$ After the effect of the supraorbital nerve block disappeared, hypoalgesia was achieved in the innervation area of the supraorbital nerve, indicating that the nerve was ablated.

\section{Data Collection}

All data were available in a patient database that contained baseline characteristics, surgical records, and follow-up data. Patients undergoing radiofrequency thermocoagulation

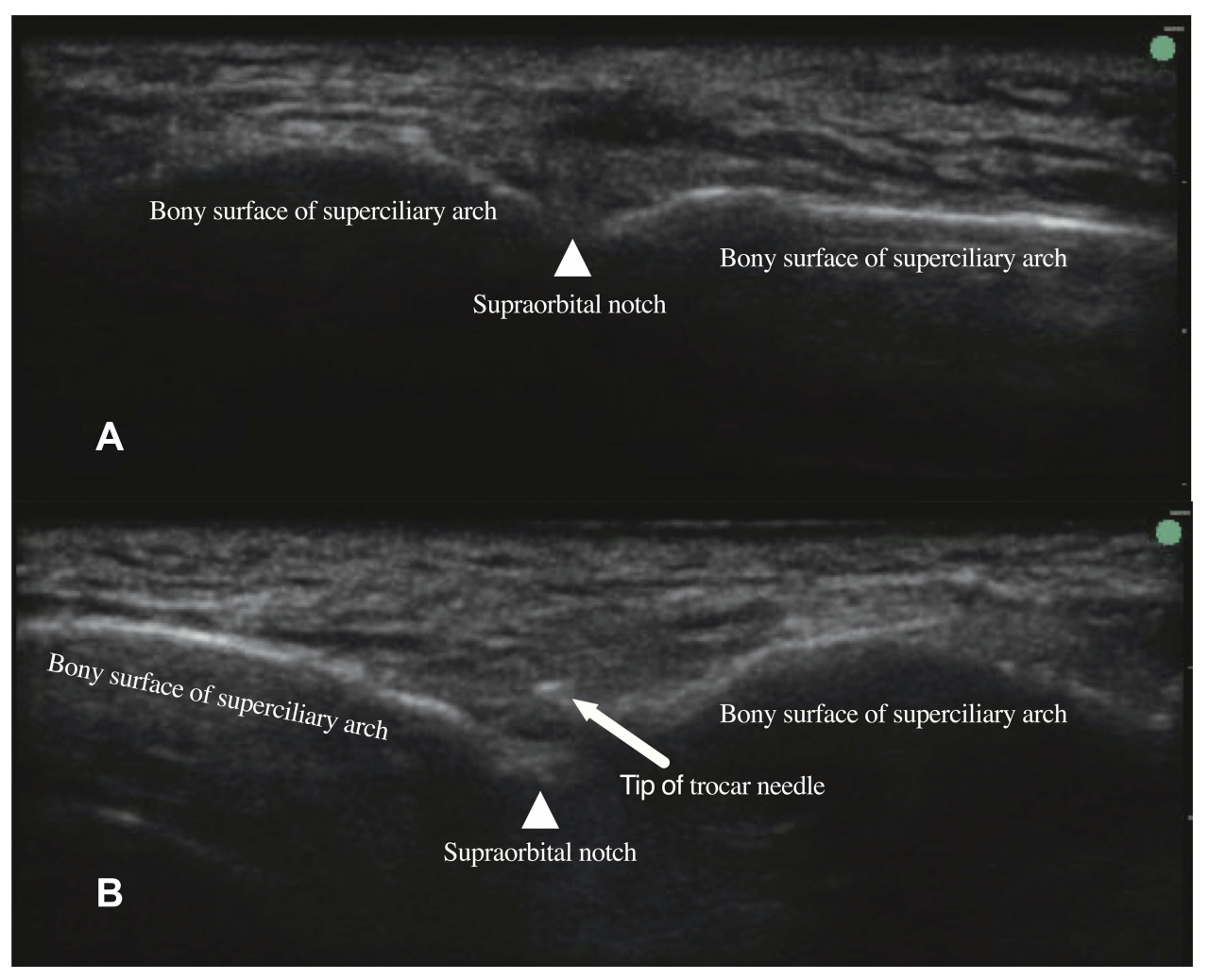

Figure I Ultrasound-guided supraorbital nerve puncture procedure.

Notes: (A) An ultrasound image of the supraorbital notch (white triangle). The high frequency linear transducer was moved from the forehead caudally to locate the supraorbital notch (white triangle), which is a small groove on the bony surface; (B) ultrasonography imaging showing that the tip of the radiofrequency treatment trocar needle (white arrow) has reached the supraorbital notch (white triangle) via the out-of-plane approach. 
in our department were routinely followed up at 1 month, 3 months, and 6 months after the procedure, followed by an annual follow-up for medical quality management. The pain intensity was assessed using a numeric rating scale (NRS) ${ }^{32}$ with 0 representing no pain and 10 representing the most severe pain imaginable. Patients could also actively report their status, such as pain recurrence or other abnormalities, over the telephone or via instant messaging. Patients with pain recurrence could also go to the pain clinic of our hospital for further examination and treatment.

We began to collect data after receiving approval from the institutional review board. Eligible patients were identified according to the inclusion and exclusion criteria. Demographic data, including age, age at onset, duration of disease, and sex, and baseline characteristics of supraorbital neuralgia, including laterality, history of forehead trauma or compression, previous treatments and preoperative pain intensity, were collected. The time of onset as well as the postoperative pain intensity at 1 month, 3 months, 6 months and annually after the procedure were also collected. The initial effective rate was defined as the percentage of patients who had an NRS score less than $50 \%$ of that before treatment within one month. The time of recurrence and subsequent treatment were collected. Recurrence was defined as an NRS score greater than $50 \%$ of that before treatment without drugs.

Data regarding complications and side effects, such as swelling or ecchymosis, numbness, and anaesthesia dolorosa, were collected. The Barrow Neurological Institute (BNI) facial numbness score ${ }^{33}$ (1: no forehead numbness; 2: mild forehead numbness, not bothersome; 3: forehead numbness, somewhat bothersome; 4: forehead numbness, very bothersome) was used to describe the symptomatic extent of forehead numbness at 1 month, 3 months, 6 months and 12 months after the procedure.

\section{Statistical Analysis}

Statistical analyses were performed using IBM SPSS Statistics software (version 25, IBM, Inc., USA). The data are reported as the mean with standard deviation, medians with interquartile ranges (IQRs), or counts and percentages according to the characteristics of the distribution. A Kaplan-Meier plot was used to present recurrencefree survival curves. For repeated measurement data, the Friedman test was used to detect changes in patient BNI facial numbness scores immediately after the procedure and at each follow-up time point. Bonferroni correction was used to correct multiple comparisons. The alpha value was set to 0.05 .

\section{Results}

Between January 2007 and April 2018, a total of 63 supraorbital neuralgia patients underwent an ultrasoundguided radiofrequency thermocoagulation procedure for the supraorbital nerve in the pain clinic of Beijing Tiantan Hospital. Ten patients were excluded according to the exclusion criteria; ultimately, 53 patients who satisfied the protocol requirements were included in this study, and their medical records were collected and analysed (Figure 2).

The demographic data and baseline characteristics of the supraorbital neuralgia patients are presented in Table 1. The mean age of the patients was $65.7 \pm 12.0$ years, the maximum age was 84 years, and the minimum age was 40 years. The mean age at onset was $58.8 \pm 12.7$ years. The median duration of disease was 6.0 years (IQR, 2.0-9.5 years; range from 0.5 years to 24 years). Of the 53 patients, $34(64.2 \%)$ were male. The disease was unilateral in all patients, and 31 cases (58.5\%) were right-sided. Eleven $(20.8 \%)$ patients could recall trauma to the brow bone or forehead, and nine (17.0\%) patients could recall compression, such as wearing goggles for a long period, in that area. All patients failed to respond to conservative treatment, including oral medicine and nerve blockade with steroids and local anaesthetics, before ultrasoundguided radiofrequency thermocoagulation treatment. Fifteen $(28.3 \%)$ patients had experienced pulsed radiofrequency treatment that was ineffective or had experienced relapse. All patients had severe pain, with a median NRS score of 8 points (IQR, 8-9 points).

All patients were completely pain free immediately after the procedure due to the effect of the nerve block with local anaesthetics. Once the effects of the local anaesthetics subsided, all the patients were examined for hypoalgesia in the supraorbital innervation area on the affected side. Forty-eight patients (90.6\%) experienced an immediate significant reduction in the NRS scores after the procedure, and the dose of the preoperative drug was gradually decreased. In 5 patients $(9.4 \%)$, there was no significant decrease in NRS scores immediately after the procedure, and these patients needed to continue treatment with oral analgesics. The pain began to gradually decrease at an average of 7 days (range 3 to 14 days) after the procedure. All patients achieved complete pain relief within one month (the initial effective rate was 100\%) 


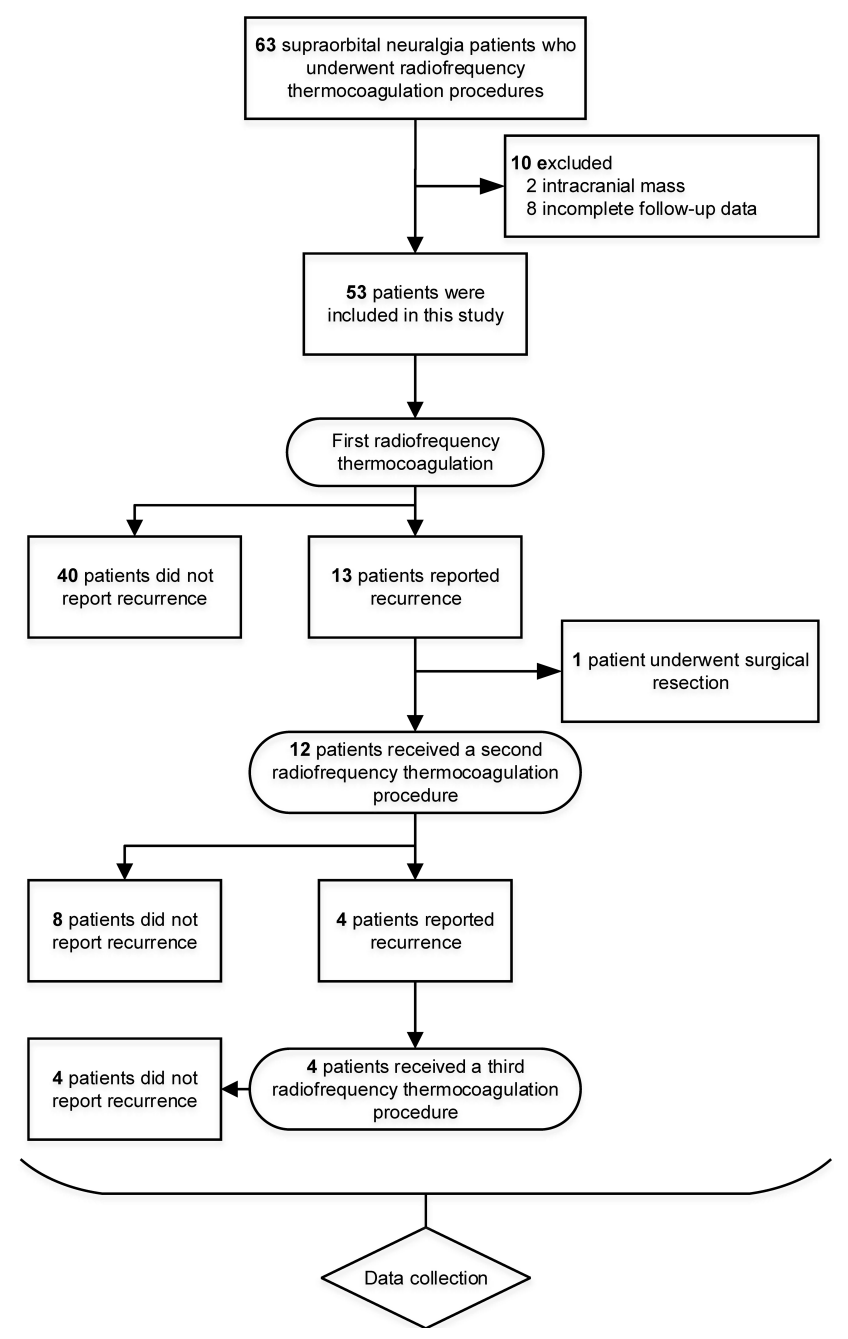

Figure 2 Flow diagram of the study populations.

Notes: A total of sixty-three supraorbital neuralgia patients underwent an ultrasound-guided radiofrequency thermocoagulation procedure. Fifty-three patients who satisfied the protocol requirements were included in this study. Thirteen patients had pain recurrence, and twelve of them received repeated radiofrequency thermocoagulation. Four of the twelve patients relapsed again, and a third radiofrequency thermocoagulation procedure was performed.

after the procedure and stopped taking drugs. The median follow-up time of the 53 patients was 36.0 months (IQR, 12.0-72.0 months). A total of 13 patients (24.5\%) had pain recurrence, with a median recurrence-free time of 97 months according to the Kaplan-Meier estimator (Figure 3). The 13 patients experienced recurrence at 9, $12,15,21,22,36,36,37,40,47,48,60$, and 97 months after the procedure. The cumulative proportion of patients with recurrence-free survival was $96.2 \%$ at 12 months, $88.4 \%$ at 24 months, $82.7 \%$ at 36 months, $70.0 \%$ at 48 months, $66.3 \%$ at 60 months, and $49.7 \%$ at 97 months.

Among the patients with recurrent pain, one underwent surgical resection of the supraorbital nerve due to cardiac pacemaker insertion after the previous radiofrequency
Table I Demographic Data and Baseline Characteristics of the Supraorbital Neuralgia Patients

\begin{tabular}{|l|l|}
\hline & $\mathbf{n = 5 3}$ \\
\hline Age (years, mean \pm SD) & $65.7 \pm 12.0$ \\
Age at onset (years, mean \pm SD) & $58.8 \pm 12.7$ \\
Duration of disease [years, median (IQR)] & $6.0(2.0-9.5)$ \\
Sex (male, \%) & $34(64.2 \%)$ \\
Laterality (right, \%) & $3 \mathrm{I}(58.5 \%)$ \\
History of forehead trauma (n, \%) & II (20.8\%) \\
History of forehead compression (n, \%) & $9(17.0 \%)$ \\
Previous treatment of pulsed radiofrequency (n, \%) & I5 (28.3\%) \\
Preoperative numeric rating scale [0-I0, median & $8(8-9)$ \\
(IQR)] & \\
\hline
\end{tabular}

thermocoagulation procedure, which is a contraindication to radiofrequency therapy. The remaining 12 patients underwent repeated radiofrequency thermocoagulation of the supraorbital nerve. Complete pain relief was achieved in all relapsed patients, regardless of receiving either radiofrequency thermocoagulation or surgical resection. For the second radiofrequency thermocoagulation treatment, the follow-up time of patients ranged from 11 to 72 months (median, 25.5 months; IQR, 13.8-40.5 months). Four patients relapsed again and underwent a third radiofrequency thermocoagulation procedure. Pain was relieved after the third procedure. As of the last available data, these four patients were followed up for $6,12,24$ and 36 months, and no pain recurrence was observed.

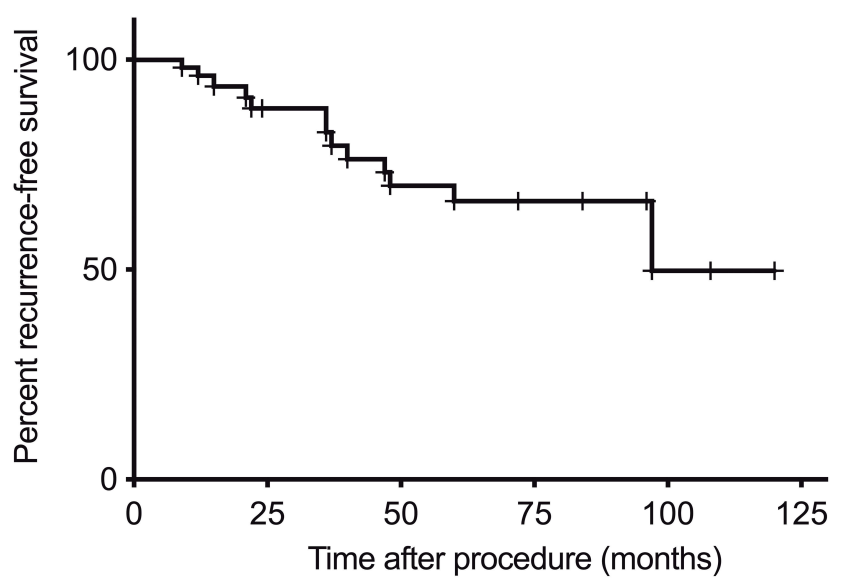

Figure 3 Kaplan-Meier recurrence-free survival curves for supraorbital neuralgia patients who underwent ultrasound-guided radiofrequency thermocoagulation procedures.

Notes: The median follow-up time of the 53 patients was 36.0 months (IQR, 12.0-72.0 months). A total of 13 patients experienced pain recurrence with a median recurrence-free time of 97 months. $X$-axis: follow-up time after procedure (months); Y-axis: the cumulative proportion of recurrence-free survival; +censoring. 
The short-term postoperative complications were eyelid and eyebrow arch swelling as well as ecchymosis, all of which could disappear within 2-3 weeks. Numbness of the supraorbital nerve innervation (forehead) occurred in all patients after the procedure. The BNI facial numbness score for the supraorbital nerve innervation of the patients is shown in Figure 4. For the BNI facial numbness score, 53 patients completed the first 6 months of follow-up, and 52 patients completed 12 months of follow-up (one patient relapsed 9 months after the procedure and was retreated with radiofrequency thermocoagulation). Out of all 53 patients, $92.5 \%$ had a BNI facial numbness score of 3 or 4 points immediately after the procedure, and the score was $81.1 \%$ at 1 month after the procedure. The numbness scores gradually decreased over time. However, of the 52 patients who completed the 12-month follow-up, 22 $(42.3 \%)$ and $3(5.6 \%)$ patients complained of numbness with scores of 3 and 4 points, respectively, at one year

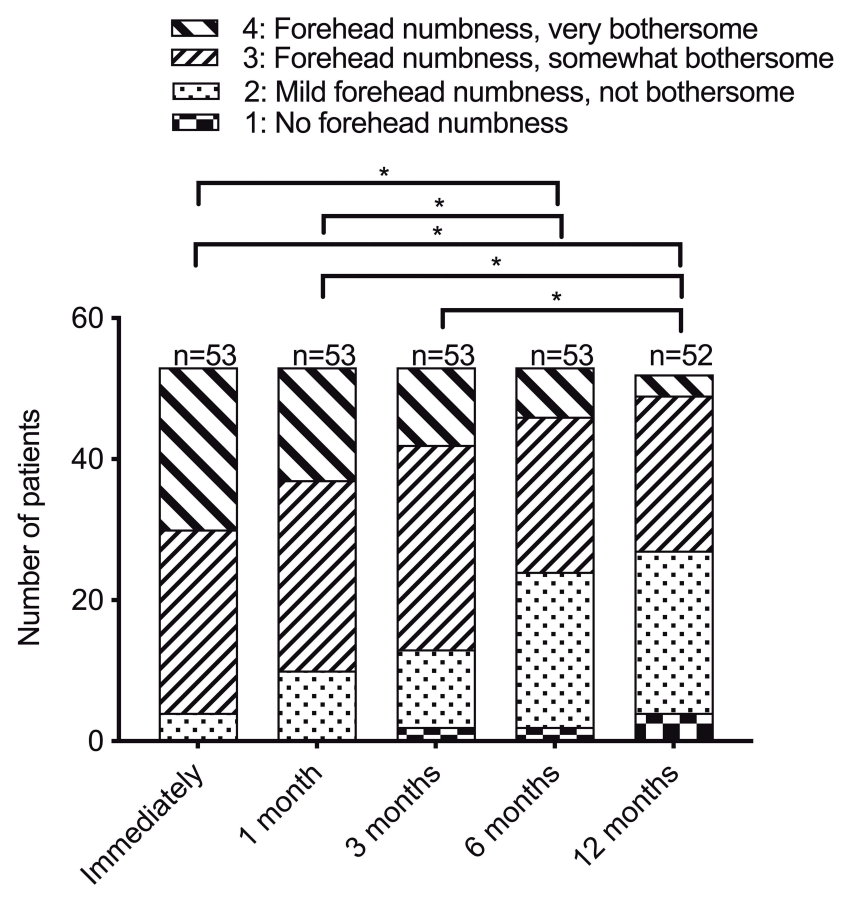

Time after procedure

Figure 4 The BNI facial numbness score for the supraorbital nerve innervation. Notes: Regarding the BNI facial numbness score, 53 patients completed the first 6 months of follow-up, and 52 patients completed 12 months of follow-up (one patient relapsed 9 months after the procedure and was retreated with radiofrequency thermocoagulation). The Friedman test was performed in 52 patients who completed the one-year follow-up. Bonferroni correction was used to correct multiple comparisons. The numbness scores at 6 and 12 months after the procedure were significantly different from those immediately and I month after the procedure; the numbness scores at 12 months after the procedure were also significantly different from those 3 months after the procedure. $* \mathrm{P}<0.05 /$ $10=0.005$. X-axis: follow-up time after procedure (months); Y-axis: number of patients. after the procedure. BNI facial numbness score data from 52 patients who completed the 12-month follow-up were included in the Friedman test. The numbness scores at 6 and 12 months after the procedure were significantly different from those immediately and 1 month after the procedure; the numbness scores at 12 months after the procedure were also significantly different from those 3 months after the procedure according to the Friedman test $(\mathrm{n}=52$, Bonferroni corrected $\alpha=0.05 / 10)$. No patients reported anaesthesia dolorosa. There were no serious adverse events, such as corneal injury, keratitis, blindness, or infection.

\section{Discussion}

As the current study shows, ultrasound-guided radiofrequency thermocoagulation for supraorbital neuralgia can provide a promising pain relief effect with an initial effective rate of $100 \%$. Ducic et al reported a series of six patients who underwent surgical resection of the supraorbital nerve, and five patients exhibited satisfactory pain relief. ${ }^{17}$ The high initial effective rate of radiofrequency thermocoagulation in this study seems to be consistent with surgical resection since these techniques are neurologically damaging procedures that can attain analgesic effects by blocking the transmission of pain. However, radiofrequency thermocoagulation is easier to perform and causes less trauma than surgical resection. For the non-destructive procedure, we previously reported that the initial effective rate of pulsed radiofrequency for supraorbital neuralgia was $77 \% .^{22}$ Amin et al reported a case series of peripheral nerve stimulation in which 16 patients underwent a trial of supraorbital peripheral nerve stimulation and 11 patients reported an improvement in pain. ${ }^{21}$ Compared with non-destructive procedures, such as pulsed radiofrequency or peripheral nerve stimulation, radiofrequency thermocoagulation achieves a better initial effective rate. Surgical decompression is another nondestructive procedure. In a case series study, pain was successfully relieved in a total of 5 patients with supraorbital neuralgia after supraorbital nerve decompression. ${ }^{20}$ However, little quantitative analysis of the efficacy of the decompression procedure is available. We assume that the high initial effective rate of radiofrequency thermocoagulation for supraorbital neuralgia observed in our study might be attributable to the ultrasound-guided percutaneous approach used. This visualization technique ensures the accuracy of the puncture position. Moreover, for a small number of patients in our study, satisfactory effects 
could not be achieved immediately after the treatment, suggesting that a short recovery period may be needed. The reason for the delayed effect may be related to the local puncture and heat damage. Therefore, in clinical practice, patients with unsatisfactory pain relief after radiofrequency thermocoagulation for supraorbital neuralgia should continue to be treated with drugs for a period and should choose other treatments after confirming that the radiofrequency thermocoagulation procedure was ineffective.

We collected long-term follow-up data for supraorbital neuralgia patients treated using radiofrequency thermocoagulation. The median follow-up duration of this case series was 36.0 months. Govind et al noted that unlike radiofrequency thermocoagulation for trigeminal neuralgia, which damages the cell bodies in the ganglion, pain might be more likely to recur with radiofrequency thermocoagulation for supraorbital neuralgia as the nerve could regenerate after peripheral nerve ablation in theory. ${ }^{34}$ Nevertheless, in our case series, radiofrequency thermocoagulation for supraorbital neuralgia maintained longterm pain relief. The median recurrence-free time was 97 months. In our previous report, for all 22 supraorbital neuralgia patients receiving pulsed radiofrequency treatment, the overall effective rate at two years postoperatively decreased to $50 \%$, and 6 of 17 patients relapsed within two years. ${ }^{22}$ In addition, Sjaastad et al performed surgical decompression on 5 cases, and two patients relapsed within two years after surgery. ${ }^{20}$ However, for the ultrasound-guided radiofrequency thermocoagulation procedure used in this study, the cumulative proportion of recurrence-free survival was $88.4 \%$ at 2 years after the procedure. This discrepancy could be attributable to the different mechanisms of treatment. Perhaps this neurological damage could provide longer pain relief. Of course, prospective randomized controlled studies are still needed to obtain a higher level of evidence.

Moreover, radiofrequency thermocoagulation can be performed repeatedly after pain recurrence. ${ }^{34}$ In this study, all patients with pain recurrence who underwent a second or third radiofrequency thermocoagulation procedure still achieved complete pain relief. Although accurately assessing the periods of pain relief after repeated procedures for insufficient numbers of patients is difficult, it did not appear to have a significantly diminishing effect. The pain relief duration of supraorbital nerve radiofrequency thermocoagulation and the effect of repeated treatments require further evaluation.
Accurate localization of the supraorbital nerve is the key point to ensure the therapeutic effects of radiofrequency thermocoagulation. In our study, all radiofrequency thermocoagulation procedures were performed under ultrasound guidance; therefore, the accuracy of puncture reached $100 \%$. Ultrasonography is a simple, noninvasive tool with no ionizing radiation that indirectly locates the supraorbital nerve by showing the hypoechoic break in the bony surface and the supraorbital artery, thus guiding the radiofrequency treatment trocar needle to approach the supraorbital nerve and avoiding damage to surrounding tissue, such as the eyeball. ${ }^{22,35}$ The accuracy of ultrasound-guided blocks of the supraorbital and infraorbital nerves is greater than that of landmark-based techniques, according to a cadaveric study. ${ }^{36}$ Ultrasonography may also detect nerve entrapment. ${ }^{37}$ Although the supraorbital nerve is well positioned by the supraorbital notch in most cases, some patients may have anatomical variations in the supraorbital structures. ${ }^{38}$ Therefore, the detection of possible anatomical variations by ultrasound is of great significance to improve the therapeutic efficiency of radiofrequency thermocoagulation.

Only mild and short-term recoverable swelling and ecchymosis occurred after the procedure. The results of this study indicate that ultrasound-guided radiofrequency thermocoagulation of the supraorbital nerve is a safe procedure with minimal side effects. This result is consistent with that of other percutaneous techniques for the treatment of supraorbital neuralgia. ${ }^{18,22,35,39}$ The most important and predictable side effect is numbness, which is caused by the disconnection of the supraorbital nerve. The degree of numbness will gradually decrease over time. However, a considerable number of patients still have numbness one year after the procedure. We speculate that the decrease in the BNI facial numbness score may be due to the patient's adaptation to numbness or to potential nerve repair. There is a lack of objective assessments of nerve damage and repair in this study. A prospective study is needed to explore the relationship between a reduced BNI facial numbness score and relapse. This issue is a limitation of radiofrequency thermocoagulation and may be the reason why some patients are reluctant to receive this therapy. In contrast, non-destructive procedures, such as pulsed radiofrequency, peripheral nerve stimulation and surgical decompression, do not cause numbness in the forehead. ${ }^{20-22,40-42}$ Therefore, as a minimally invasive percutaneous interventional technique, non-destructive pulsed radiofrequency can be 
a treatment option before radiofrequency thermocoagulation treatment. However, this strategy will also increase the risk of surgery and medical costs. Pulsed radiofrequency combined with radiofrequency thermocoagulation at a lower temperature may also be a treatment with maximum efficacy and minimum side effects. ${ }^{43,44}$ Thus, the optimal strategy for supraorbital neuralgia remains to be further explored.

This study has several limitations. First, this is a case series report without a control group; therefore, the evidence and conclusions of this study are not strong. Second, retrospective studies and the inclusion of patients over a large time span may lead to potential bias; for example, the diagnostic criteria may not be uniform. Third, because the incidence of supraorbital neuralgia is low and most patients do not need to undergo a destructive procedure, the sample size of this study is small, and its statistical power may be insufficient. Fourth, the data were collected during routine clinical follow-up, and the frequency of follow-up was low. Thus, the follow-up may have been inadequate for detecting pain recurrence in time. Fifth, the evaluation of pain in the study was based solely on the NRS and lacked objective evaluation indicators such as pain-related evoked potentials. Sixth, the BNI facial numbness score is used to evaluate the trigeminal neuralgia treatment procedure, not the supraorbital neuralgia treatment. This study lacked an objective assessment of numbness and nerve repair. Moreover, the assessment was limited to 12 months after the procedure, which was not long enough to explain the relationship between decreased BNI facial numbness score and pain recurrence. Seventh, our study included patients with either traumatic or nontraumatic supraorbital neuralgia who may have had different responses to radiofrequency thermocoagulation.

\section{Conclusion}

This study indicates that ultrasound-guided radiofrequency thermocoagulation is an effective and safe treatment for supraorbital neuralgia patients who fail to respond to conventional therapies such as oral medicine, nerve blockade or pulsed radiofrequency. Radiofrequency thermocoagulation can be performed repeatedly after recurrence, and repeated radiofrequency thermocoagulation treatment is still effective. The most important and predictable side effect is numbness in the forehead, but this postoperative numbness can gradually decrease. Prospective, randomized, controlled clinical studies are necessary to provide higher levels of evidence regarding the efficacy and safety of ultrasound-guided radiofrequency thermocoagulation for the treatment of supraorbital neuralgia patients who respond poorly to conservative treatments.

\section{Acknowledgments}

This work was supported by the Beijing Municipal Administration of Hospitals Clinical Medicine Development of Special Funding Support (grant No. XMLX201707) and the Foundation for the Excellent Medical Staff of Beijing (grant No. 2014-3-035).

\section{Disclosure}

The authors report no conflicts of interest in this work.

\section{References}

1. Standring S. Face and scalp. In: Standring S, editor. Gray's Anatomy. 41 ed. Elsevier; 2016:475-506.

2. Sjaastad O, Petersen HC, Bakketeig LS. Supraorbital neuralgia. Vaga study of headache epidemiology. Cephalalgia. 2005;25(4):296-304. doi:10.1111/j.1468-2982.2004.00856.x

3. Arnold M. Headache classification committee of the International Headache Society (IHS) the international classification of headache disorders, 2nd edition. Cephalalgia. 2004;24(Suppl. 1):1-160.

4. Arnold M. Headache classification committee of the International Headache Society (IHS) the international classification of headache disorders, 3rd edition. Cephalalgia. 2018;38(1):1-211. doi:10.1177/ 0333102417738202

5. World Health Organization. International statistical classification of diseases and related health problems (11th Revision). https://icd.who. int/browse11/1-m/en. Published 2018. Accessed 2019.

6. Waldman SD. Supraorbital neuralgia. In: Waldman SD, editor. 3, trans. Atlas of Uncommon Pain Syndromes. Philadelphia: Elsevier; 2014:3-6.

7. Caminero AB, Pareja JA. Supraorbital neuralgia: a clinical study. Cephalalgia. 2001;21(3):216-223. doi:10.1046/j.1468-2982.2001.0 0190.x

8. Mulero P, Guerrero AL, Pedraza M, et al. Non-traumatic supraorbital neuralgia: a clinical study of 13 cases. Cephalalgia. 2012;32 (15):1150-1153. doi:10.1177/0333102412459575

9. Jacobson RI. More "goggle headache": supraorbital neuralgia. $N$ Engl $J$ Med. 1983;308(22):1363.

10. De Giorgis M. Supraorbital neuralgia: a benign condition. Eur J Pain Suppl. 2010;4(1):65-66. doi:10.1016/S1754-3207(10)70229-7

11. Stewart M, Boyce S, McGlone R. Post-traumatic headache: don't forget to test the supraorbital nerve! BMJ Case Rep. 2012. doi:10.1136/bcr-2012-006936

12. Penas-Prado M, Martinez-Salio A, Porta-Etessam J, et al. Posttraumatic supraorbital neuralgia: a benign condition. Rev Neurol. 2007;44(2):89-91.

13. Evans RW, Pareja JA. Expert opinion. Supraorbital neuralgia. Headache. 2009;49(2):278-281. doi:10.1111/hed.2009.49.issue-2

14. Pareja JA, Caminero AB. Supraorbital neuralgia. Curr Pain Headache Rep. 2006;10(4):302-305. doi:10.1007/s11916-006-0036-9

15. Jadhav V, Patil D, Mane M. Supraorbital neuralgia. Med J Dr DY Patil Vidyapeeth. 2014;7(2):208. doi:10.4103/0975-2870.126346

16. Wilkinson HA. Trigeminal nerve peripheral branch phenol/glycerol injections for tic douloureux. J Neurosurg. 1999;90(5):828-832. doi:10.3171/jns.1999.90.5.0828

17. Ducic I, Larson EE. Posttraumatic headache: surgical management of supraorbital neuralgia. Plast Reconstr Surg. 2008;121(6):1943-1948. doi:10.1097/PRS.0b013e3181707063 
18. Trescot AM. Cryoanalgesia in interventional pain management. Pain Physician. 2003;6(3):345-360.

19. Justiz R, Trescot AM. Supraorbital nerve entrapment. In: Trescot AM, editor. Peripheral Nerve Entrapments: Clinical Diagnosis and Management. Cham: Springer International Publishing; 2016:95-104.

20. Sjaastad O, Stolt-Nielsen A, Pareja JA, Fredriksen TA, Vincent M. Supraorbital neuralgia. On the clinical manifestations and a possible therapeutic approach. Headache. 1999;39(3):204-212. doi:10.1046/ j.1526-4610.1999.3903204.x

21. Amin S, Buvanendran A, Park KS, Kroin JS, Moric M. Peripheral nerve stimulator for the treatment of supraorbital neuralgia: a retrospective case series. Cephalalgia. 2008;28(4):355-359. doi:10.1111/j.1468-2982.2008.01535.x

22. Luo F, Lu J, Ji N. Treatment of refractory idiopathic supraorbital neuralgia using percutaneous pulsed radiofrequency. Pain Pract. 2018;18:871-878. doi:10.1111/papr.2018.18.issue-7

23. Beyer T. Idiopathic supraorbital neuralgia. Laryngoscope. 1949;59 (11):1273-1275. doi:10.1288/00005537-194911000-00007

24. Vanelderen P, Rouwette T, De Vooght P, et al. Pulsed radiofrequency for the treatment of occipital neuralgia a prospective study with 6 months of follow-up. Reg Anesth Pain Med. 2010;35(2):148-151. doi:10.1097/AAP.0b013e3181d24713

25. Chua NHL, Vissers KC, Sluijter ME. Pulsed radiofrequency treatment in interventional pain management: mechanisms and potential indications-a review. Acta Neurochir (Wien). 2011;153(4):763-771. doi:10.1007/s00701-010-0881-5

26. Lan M, Zipu J, Ying S, Hao R, Fang L. Efficacy and safety of CT-guided percutaneous pulsed radiofrequency treatment of the Gasserian ganglion in patients with medically intractable idiopathic trigeminal neuralgia. J Pain Res. 2018;11:2877-2885. doi:10.2147/JPR

27. Kanpolat Y, Savas A, Bekar A, Berk C. Percutaneous controlled radiofrequency trigeminal rhizotomy for the treatment of idiopathic trigeminal neuralgia: 25-year experience with 1600 patients. Neurosurgery. 2001;48(3):524-532. [discussion 532-524]. doi:10.1097/00006123-200103000-00013

28. Kim JH, Yu HY, Park SY, Lee SC, Kim YC. Pulsed and conventional radiofrequency treatment: which is effective for dental procedure-related symptomatic trigeminal neuralgia? Pain Med. 2013;14(3):430-435. doi:10.1111/pme. 12046

29. Li X, Ni J, Yang L, et al. A prospective study of Gasserian ganglion pulsed radiofrequency combined with continuous radiofrequency for the treatment of trigeminal neuralgia. J Clin Neurosci. 2012;19 (6):824-828. doi:10.1016/j.jocn.2011.07.053

30. Yao P, Hong T, Wang ZB, et al. Treatment of bilateral idiopathic trigeminal neuralgia by radiofrequency thermocoagulation at different temperatures. Medicine (Baltimore). 2016;95(29):e4274. doi:10.1097/ MD.0000000000004274

31. Nie F, Su D, Shi Y, et al. A prospective study of X-ray imaging combined with skin stimulation potential-guided percutaneous radiofrequency thermocoagulation of the Gasserian ganglion for treatment of trigeminal neuralgia. Pain Med. 2014;15(9):1464-1469. doi:10.1111/pme.12359
32. Ferreira-Valente MA, Pais-Ribeiro JL, Jensen MP. Validity of four pain intensity rating scales. Pain. 2011;152(10):2399-2404. doi:10.1016/j.pain.2011.07.005

33. Rogers CL, Shetter AG, Fiedler JA, Smith KA, Han PP, Speiser BL. Gamma knife radiosurgery for trigeminal neuralgia: the initial experience of the barrow neurological institute. Int J Radiat Oncol Biol Phys. 2000;47(4):1013-1019. doi:10.1016/S0360-3016(00)00513-7

34. Govind J, King W, Bailey B, Bogduk N. Radiofrequency neurotomy for the treatment of third occipital headache. J Neurol Neurosurg Psychiatry. 2003;74(1):88-93. doi:10.1136/jnnp.74.1.88

35. Lee JY, Sim WS, Kim DK, Park HJ, Oh MS, Lee JE. Ultrasoundguided pulsed radiofrequency treatment for postherpetic neuralgia of supraorbital nerve: a case report. Anesth Pain Med. 2014;9 (2):103-105.

36. Spinner D, Kirschner JS. Accuracy of ultrasound-guided superficial trigeminal nerve blocks using methylene blue in cadavers. Pain Med. 2012;13(11):1469-1473. doi:10.1111/j.1526-4637.2012.01480.x

37. Tijssen C, Schoemaker K, Visser L. Supraorbital neuralgia caused by nerve entrapment visualized on ultrasonography. Headache. 2013;53 (2):376-377. doi:10.1111/head.2013.53.issue-2

38. Tomaszewska A, Kwiatkowska B, Jankauskas R. The localization of the supraorbital notch or foramen is crucial for headache and supraorbital neuralgia avoiding and treatment. Anat Rec (Hoboken). 2012;295(9):1494-1503. doi:10.1002/ar.22534

39. Lee JH, Kim TY, Ha SH, Kwon YE, Yoon CS. Pulsed radiofrequency lesioning of supraorbital and supratrochlear nerve in postherpetic neuralgia -a report of 2 cases. Korean J Pain. 2004;17(2):239. doi:10.3344/jkps.2004.17.2.239

40. Asensio-Samper JM, Villanueva VL, Perez AV, et al. Peripheral neurostimulation in supraorbital neuralgia refractory to conventional therapy. Pain Pract. 2008;8(2):120-124. doi:10.1111/j.15332500.2007.00165.x

41. Sachdeva H, Hoffman L, Abd-Elsyed A. Pulsed radiofrequency of the supraorbital nerve for the treatment of supraorbital neuralgia. Saudi J Anaesth. 2017;11(4):505-506. doi:10.4103/sja.SJA_159_17

42. Dua A, Shepherd J, Bahadur S, Martin D. Supraorbital neuralgia treatment with radio frequency ablation. J Pain. 2016;17(4):S92. doi:10.1016/j.jpain.2016.01.449

43. Ali Eissa AA, Reyad RM, Saleh EG, El-Saman A. The efficacy and safety of combined pulsed and conventional radiofrequency treatment of refractory cases of idiopathic trigeminal neuralgia: a retrospective study. J Anesth. 2015;29(5):728-733. doi:10.1007/s00540-015-2029-5

44. Elawamy A, Abdalla EEM, Shehata GA. Effects of pulsed versus conventional versus combined radiofrequency for the treatment of trigeminal neuralgia: a prospective study. Pain Physician. 2017;20 (6):E873-e881.
Journal of Pain Research

\section{Publish your work in this journal}

The Journal of Pain Research is an international, peer reviewed, open access, online journal that welcomes laboratory and clinical findings in the fields of pain research and the prevention and management of pain. Original research, reviews, symposium reports, hypothesis formation and commentaries are all considered for publication. The manuscript

Submit your manuscript here: https://www.dovepress.com/journal-of-pain-research-journal management system is completely online and includes a very quick and fair peer-review system, which is all easy to use. Visit http:// www.dovepress.com/testimonials.php to read real quotes from published authors. 